\title{
Scanning the Issue
}

\section{Special Issue on Power Technology and Policy: Forty Years After the 1965 Blackout}

This issue concerns the evolving needs, technologies, and industry structures of the electric power industry. While the ultimate objective of this industry remains the providing of a sustainable high quality of service (QoS) to its users, the needs for this service have become more complex than has been the case in the past. This complexity comes from a peculiar mixture of nonuniform user needs, disruptive technologies, and regulatory restructuring.

Concerning the needs, it is fair to say that customers still want continuous abundant cost-effective service. As a minimum, customers and the society as a whole should be shielded against basic service interruption during unplanned cascading equipment failures, such as that experienced during the recent blackouts in the United States, Western Europe, and Russia. At the same time, it is becoming increasingly clear that the differentiation of QoS is technically viable and of potential interest. The measures of QoS are multiple, and range from the rate and duration of service interruptions; through voltage and frequency deviations from nominal; the willingness to sign up for programs offering lower prices according to well-defined terms of service; and, finally, the willingness to pay more for being served from clean environmentally sustainable energy sources.

It is well known to the IEEE community that electrification was one of the biggest technological achievements of the past century. Much progress in the society as a whole has rested on this achievement. The design and operations of Nikola Tesla's far reaching vision of three-phase ac transmission network systems have provided the basis for producing and delivering electricity over large distances and to many users. Thomas Edison's vision of dc smaller-scale distributed electric power systems close to the end users remains an important alternative. For a very long time the bulk electric power has been managed by stepping up ac voltage of power produced by the very large synchronous generators to the EHV backbone transmission grid, and transmitting bulk power to the load centers, and stepping down to the utility HV subtransmission and MV/LV local distribution networks all the way to the smallest users. This

Digital Object Identifier 10.1109/JPROC.2005.857495 architecture was mainly determined by economic considerations which favored building large equipment rather than small scale energy sources. More recently there have been many breakthroughs in disruptive distributed hardware and software technologies which may lead to qualitatively different architectures and operating paradigms, notably an active adaptation by the end users. In contrast with today's top-down planning and management approach, these new technologies could enable distributed decision making by the customers, utilities, and regions, with minimal coordination at the interconnection level. Moving toward such multilayered flexible provision and use of electricity services poses a considerable challenge.

The recent blackouts have raised questions concerning electric power grid reliability and possible new avenues for its improvement. While these questions are becoming increasingly more complex as the electric power industry restructures, a worthwhile task is first to assess current engineering practices and second to identify avenues for improvement in the regulated industry. This process is particularly critical in order to understand the costs and benefits related to a variety of engineering proposals made following the blackouts. We have been presented with a range of long-term technologies, extremely expensive to develop, as well as with technologies which could be very useful and cost-effective in the near future. While the basic potential for technological progress in the electric power industry is huge, an assessment of the different solutions prior to adopting specific technologies is essential. The recently approved U.S. Energy Bill includes active measures for forming an Electricity Reliability Organization (ERO) responsible for ensuring that, as changes take place, service to the customers remains constant.

Concerning regulatory restructuring, the most recent blackouts have brought public attention to the need to understand the interdependence between policy, economic, financial, and engineering developments. Understanding this interdependence is essential because, over the past ten years or so, we have witnessed only piecemeal efforts to change the electric power industry. In particular, the industry restructuring which has occurred has been a scattered process that has taken place without a firm vision of an 
ultimate goal and with very little understanding of what is realistically possible. The issues have been approached primarily as short-term political matters instead of genuine systems engineering problems. The outcomes have caught many by surprise. This special issue is motivated by the overall situation described above. The papers are written with the intent to provide an analysis of the major issues and challenges underlying possible solutions.

\section{COVERAGE}

The first part of this issue is devoted to assessing the operations of large-scale electric power interconnections, in particular the technological enhancements needed to manage these in a more predictable way during large equipment outages. The papers by $\mathrm{Wu}$ et al. and by Kezunović and Abur concern the communications and information protocols, while the papers by Ilić et al. and by Makarov et al. concern advances in real-time control. The paper by $\mathrm{Wu}$ et al. reviews the functions and architectures of control centers: their past, present, and likely future. The paper points out that a great impetus to the development of control centers occurred after the Northeast blackout of 1965 when it was recommended that computers be deployed to improve the real-time operations of the interconnected system. The paper points out that over the years, information technologies have progressed in leaps and bounds, while control centers, with their nonstandard legacy devices and systems that could not take full advantage of the new technologies, have remained largely unchanged. Qualitative changes are described that would make control centers decentralized, yet still integrated, flexible, and open. The paper by Kezunović and Abur is closely related to the $\mathrm{Wu}$ et al. paper in its quest to merge the temporal and spatial aspects of data and information for improved power systems monitoring. As an illustration, two new monitoring functions are discussed: 1) automated fault analysis; and 2) hierarchical state and topology estimation. The paper by Ilić et al. concerns the critical role enhanced control will play in the operating of future electric power systems. A multilayered approach is introduced to support: 1) on-line adjustment of available resources; 2) monitoring of the interconnection based on indices essential for deciding the severity of the operating mode; and 3) the use of these indices to adjust the control structure as the system evolves from one mode to the next. The paper by Makarov et al. concerns recent blackouts in the United States, Europe, and Russia. The paper advocates the urgent need for adequate research and development to investigate cascading processes, determine the conditions and triggering events that cause blackouts, and develop preventive methods. The paper summarizes the main principles and elements of the Russian blackout prevention system that authors suggest may not be well-known elsewhere.

The second part of this issue provides an assessment of major design and operational challenges that could hamper reliable electricity service in the changing industry. While it is often difficult to separate strict engineering complexities from those induced by regulatory changes, one fact is certain: providing reliable and efficient service has become more complicated because regulatory decisions directly affect the grid requirements for system support needed to implement energy trades. In addition to the problems perceived by the market participants, one must consider the engineering problem of active exchange of power across the control areas at levels for which the system was not initially designed. Trading requirements present the industry with serious engineering challenges if the trading is to be done without reliability problems. The paper by Chow et al., in particular, makes the case that system reliability is an integral part of a properly designed electricity market, even though wholesale prices are its most visible part. A vision of an integrated approach to ensuring reliable service within a market is offered. The paper suggests that cooptimization of energy and reserves is a more effective way of providing reliable service than having separate sub-markets for reserves. The paper by Galiana et al. is synergic with the Chow et al. paper in the sense that a single market for cooptimizing energy and reserves is suggested instead of sequential provision by several sub-markets. An example of joint market clearing of energy with reserves is provided as an illustration. Papers by Chao et $a l$. and by Baldick et al. consider the effect of market designs on: 1) overall system efficiency; 2) the ability of market participants to exercise market power by profiting in unjustifiable ways; and 3) the technical performance of the system. Chao et al., in particular, offer principles essential for designing a systematic interface between the engineering and market functions. This paper describes much more decentralization and separation of functions than the papers by Chow et al. and Galiana et al.

The third part of this issue concerns the longer term performance of electric power systems. The paper by Shahidepour et al. reviews the status of security analysis, with an emphasis on mid-term operation planning and long-term planning. The paper stresses the numerical methods for planning. The paper by Thomas et al. points out the fundamental difficulties with planning that exist in the changing electric power industry, and it analyzes solutions proposed and implemented in Australia, in particular. The paper by Roark et al. highlights the types of physical and financial uncertainties present when decisions are being made in competitive electricity markets. The paper offers an interesting illustration of various risks and the mechanisms for their management in a simple power system example. The paper by Wangensteen et al. provides an overview of markets in Northern Europe. Finally, the paper by Kadoya et al. applies a system dynamics approach to a model structure that is suitable for longer term analysis of capacity investment and market stability. The simulations suggest that, for a realistic range of assumptions, deregulated electricity markets are substantially more cyclical than the electricity provision would have been under a regulated monopoly regime. This result creates the challenge of designing longer term markets in order to reduce the cycles.

In closing, we suggest that the challenges outlined in this issue should be of great relevance to readers of the PROCEEDINGS OF THE IEEE. The level of complexity in 
today's electric power systems is enormous. Several disciplines underlying operations and planning of electric power systems as a whole are essential, such as: 1) systems, control theory, and automation; 2) distributed sensors and actuators; and 3) communications, computing, and, more broadly, information technologies. A particularly unique challenge concerns the interdependencies between the physical, economic, financial, and policy systems. Critical problems facing the electric power industry have highlighted the need for multidisciplinary studies, including the interplay between economic policy design and engineering design for well-understood performance.

\section{ACKNOWLEDGMENT}

I would like to thank all Guest Editors for their help with recommending invited contributors, and with processing their share of papers; all authors and reviewers who have tremendously contributed to this special issue; and Jim Calder, Managing Editor of the Proceedings of the IEEE, his assistant Margery Meyer, and my assistant Claire Bauerle.

\author{
MARIJA ILIĆ, Guest Editor \\ Department of Electrical and Computer \\ Engineering \\ Carnegie Mellon University \\ Pittsburgh, PA 15213-3890 USA
}

JoE H. CHOw, Guest Editor

Electrical, Computer and Systems

Engineering

Rensselaer Polytechnic Institute

Troy, NY 12180-3590 USA

\author{
Francisco D. Galiana, Guest Editor \\ Department of Electrical and Computer \\ Engineering \\ McGill University \\ Montreal, QC H3A 2A7 Canada
}
Mohammad Shahidehpour, Guest Editor
Electrical and Computer Engineering Department
Illinois Institute of Technology
Chicago IL 60616 USA

\section{Robert J. ThOMAS, Guest Editor \\ Department of Electrical and Computer Engineering \\ Cornell University \\ Ithaca NY 14853 USA}

\author{
Felix F. WU, Guest Editor \\ Center for Electrical Energy Systems \\ University of Hong Kong \\ Hong Kong
}
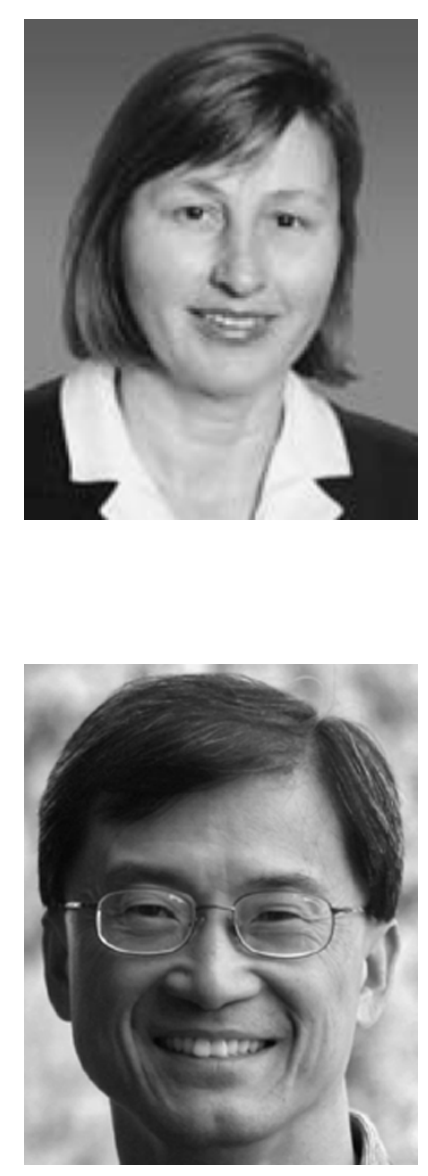

Marija Ilić (Fellow, IEEE) received the M.Sc. and D.Sc. degrees in systems science and mathematics from Washington University, St. Louis, MO, and the Dipl. Ing. and M.E.E. degrees from her native Yugoslavia, University of Belgrade.

She is a professor in the Electrical and Computer Engineering and Engineering Public Policy Departments, Carnegie-Mellon University, Pittsburgh, PA. She was a Senior Research Scientist at the EECS Department at MIT since 1987, and currently holds an Appointment of a Visiting Professor in the newly formed Engineering Systems Division at Massachusetts Institute of Technology, Cambridge. Her interest is in control and design of large-scale systems. From September 1999 until March 2001, she was a Program Director for Control, Networks and Computational Intelligence at the National Science Foundation. Prior to her years at MIT, she was a faculty at the University of Illinois at Urbana-Champaign, Cornell University, and Drexel University.

Prof. Ilić is a recipient of the First Presidential Young Investigator Award for Power Systems. She is an IEEE Distinguished Lecturer.

Joe H. Chow (Fellow, IEEE) received the M.S. and Ph.D. degrees from the University of Illinois, Urbana-Champaign, working on singular perturbation techniques.

He joined the General Electric Power System, Schenectady, NY, in 1978. He joined Rensselaer Polytechnic Institute in 1987 and is a Professor of Electrical, Computer, and Systems Engineering. He is also a consultant to NYISO. His research interests include multivariable control and power system modeling, dynamics, and control. Most recently he has been working on voltage-sourced converter-based FACTS Controllers. 

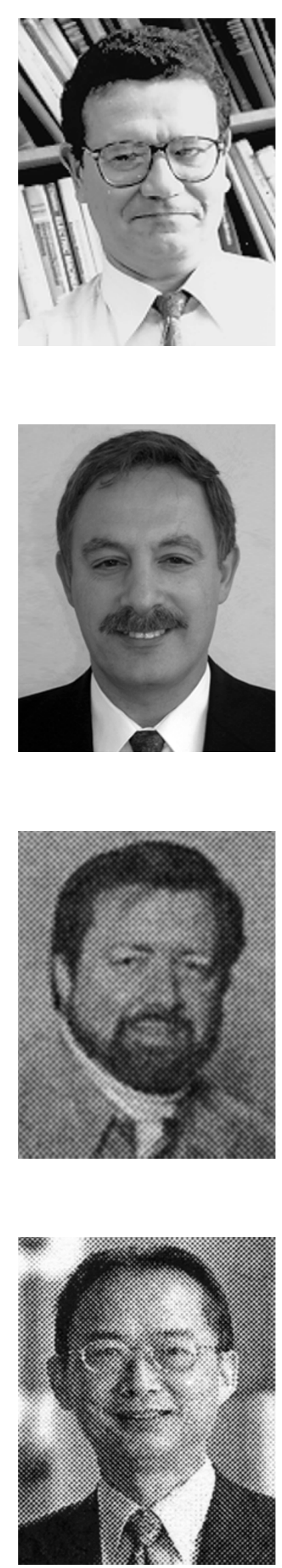

Francisco D. Galiana (Fellow, IEEE) received the B.Eng. (Hon.) degree from McGill University, Montreal, QC, Canada, in 1966 and the S.M. and Ph.D. degrees from the Massachusetts Institute of Technology, Cambridge, in 1968 and 1971, respectively.

He spent some years at the Brown Boveri Research Center, Baden, Switzerland, and held a faculty position at the University of Michigan, Ann Arbor. He joined the Department of Electrical and Computer Engineering at McGill University in 1977, where he is currently a Full Professor.

Mohammad Shahidehpour (Fellow, IEEE) is Bodine Professor and Chairman of the Electrical and Computer Engineering Department at the Illinois Institute of Technology, Chicago. He has written extensively on issues related to power systems restructuring and optimization.

Dr. Shahidehpour is the recipient of the 2005 IEEE/PES Prize Paper Award.

Robert J. Thomas (Fellow, IEEE) is currently Professor of Electrical and Computer Engineering at Cornell University, Ithaca, NY. He has served as the IEEE-USA Vice President for Technology Policy. His technical background is broadly in the areas of systems analysis and control of large-scale electric power systems. He has published in the areas of transient control and voltage collapse problems as well as technical, economic, and institutional impacts of restructuring.

Prof. Thomas has been a member of the IEEE-USA Energy Policy Committee since 1991 and was the committee's Chair from 1997 to 1998.

Felix F. Wu (Fellow, IEEE) is the Philip Wong Wilson Wong Professor in Electrical Engineering at the University of Hong Kong, Hong Kong, where he served as Pro Vice Chancellor (Vice President) from 1997 to 2001. He is also a Professor Emeritus at the University of California, Berkeley, where he has been on the faculty since 1974 . 